\title{
Overview and status of the Giant Magellan Telescope project
}

James Fanson, Patrick J. McCarthy, Rebecca Bernstein, George Angeli, David Ashby, et al.

James Fanson, Patrick J. McCarthy, Rebecca Bernstein, George Angeli, David Ashby, Bruce Bigelow, Antonin Bouchez, William Burgett, Eric Chauvin, Adam Contos, Francisco Figueroa, Peter Gray, Frank Groark, Robert Laskin, Rafael Millan-Gabet, Andrew Rakich, Rodrigo Sandoval, Marti Pi, Nune Wheeler, "Overview and status of the Giant Magellan Telescope project," Proc. SPIE 10700, Ground-based and Airborne Telescopes VII, 1070012 (6 July 2018); doi: 10.1117/12.2313340

Event: SPIE Astronomical Telescopes + Instrumentation, 2018, Austin, Texas, United States 


\title{
Overview and Status of the Giant Magellan Telescope Project
}

\author{
James Fanson*a Patrick J. McCarthy ${ }^{\mathrm{a}, \mathrm{b}}$, Rebecca Bernstein ${ }^{\mathrm{a}, \mathrm{b}}$, George Angeli ${ }^{\mathrm{a}}$, David Ashby ${ }^{\mathrm{a}, \mathrm{c}}$, \\ Bruce Bigelow ${ }^{\mathrm{a}}$, Antonin Bouchez ${ }^{\mathrm{a}}$, William Burgett ${ }^{\mathrm{a}}$, Eric Chauvin ${ }^{\mathrm{a}}$, Adam Contos ${ }^{\mathrm{a}}$, \\ Francisco Figueroa ${ }^{\mathrm{a}}$, Peter Gray ${ }^{\mathrm{a}}$, Frank Groark ${ }^{\mathrm{a}}$, Robert Laskin ${ }^{\mathrm{a}}$, Rafael Millan-Gabet ${ }^{\mathrm{a}}$, \\ Andrew Rakich ${ }^{\mathrm{a}}$, Rodrigo Sandoval ${ }^{\mathrm{a}}$, Marti $\mathrm{Pi}^{\mathrm{a}}{ }^{\mathrm{a}}$, Nune Wheeler ${ }^{\mathrm{a}}$ \\ ${ }^{\mathrm{a}}$ GMTO Corporation, 465 N. Halstead St., Suite 250, Pasadena, CA 91107, USA \\ ${ }^{\mathrm{b} C}$ Carnegie Observatories, 813 Santa Barbara St., Pasadena, CA 91101, USA \\ 'University of Arizona, 933 N. Cherry St., Tucson, AZ 85719, USA
}

\begin{abstract}
The Giant Magellan Telescope project is proceeding with design, fabrication, and site construction. The first of the seven required 8.4-m primary mirror segments is completed and in storage, three segments are in various stages of grinding and polishing, and the fifth segment has been cast. Industry contracts are underway to complete the design of the telescope structure. Residence buildings and other facilities needed to support construction at the Las Campanas site in Chile are complete. Hard rock excavation is imminent in preparation for the pouring of concrete for the telescope pier and other foundations. Computational fluid dynamics analysis is informing the design of the telescope enclosure, and further construction work packages are being readied for tender. Seismic design considerations have resulted in the incorporation of a seismic isolation system into the telescope pier, as well as modifications to the primary mirror support system. Designs for the fast-steering and adaptive secondary mirrors, science instruments, and other subsystems are maturing. Prototyping is underway in various aspects, including on-sky testing of wavefront sensing and control elements, and the telescope metrology system. Our fabrication and construction schedule calls for engineering first light with a subset of primary mirror segments in late 2023, with buildout to the full configuration occurring in stages, paced by the availability of primary mirror segments and other components.
\end{abstract}

Keywords: GMT, GMTO, Giant Magellan Telescope, Extremely Large Telescope, overview

\section{INTRODUCTION}

We provide a status report on the design and construction of the Giant Magellan Telescope. The goals of the project and the concept for the GMT have been reviewed in past proceedings of the SPIE. ${ }^{1,2,3,4,5,6}$ In this 2018 status update, we concentrate on areas of the project that have undergone significant evolution since the report in the 2016 proceedings. These include improvements in systems engineering and integrated modeling, incorporation of base isolation into the telescope pier, engagement with industry contractors for the telescope structure, advances in wavefront sensing, metrology and control, and construction on the site in Chile. We provide an update on the development of the primary optics for the telescope but note that Martin et al. in these proceedings will cover the technical aspects of this topic in greater detail. ${ }^{7} \mathrm{~A}$ significant number of papers in these proceedings address aspects of the GMT project in depth. ${ }^{8,9,10,11,12,13}$ In this overview we aim to provide a high-level view of the entire project with an emphasis on process and activities that address risk and tie together the many technical and programmatic aspects of the project.

The origins of the GMT concept, its relationship to the twin Magellan $6.5 \mathrm{~m}$ telescopes, and the motivations for the use of large primary mirror segments in a fast focal-ratio Gregorian optical design have been described in previous reports of the SPIE. ${ }^{3,5,14}$ The scientific motivations for the GMT, and all of the Extremely Large Telescopes (ELTs) under development, are stronger than ever, and recent astronomical discoveries have opened new avenues of research for the GMT and the other ELTs.

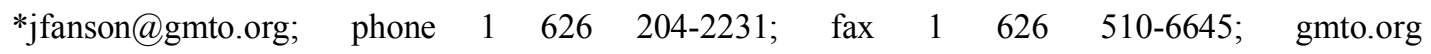


The simultaneous detection of gravitational and electromagnetic waves from merging neutron stars has opened the new field of multi-messenger astronomy. Transient signals associated with rapidly evolving phenomena (e.g. accretion events, explosions, mergers, transits) require short response times and high photon rates from large collecting areas, particularly for spectral diagnostics. The electromagnetic signal from the merging neutron stars detected with LIGO faded rapidly and was beyond the reach of spectrographs on $8 \mathrm{~m}$ telescopes in a few hours. These phenomena and the new generation of time-domain experiments have stimulated awareness of the importance of rapid time-to-target response with the GMT and its faint-object and high-resolution spectrographs. The explosive growth in our understanding of the frequency and properties of exoplanets, and rocky planets around low mass stars in particular, has led to heightened interest in studies of the atmospheric composition of exoplanets and in the potential for detection of biomarkers. The GMT consortium's high dispersion spectrographs enable these studies and related exoplanet science.

Many exoplanet science programs call for diffraction-limited images and thus rely on adaptive optics. The GMT employs adaptive secondary mirrors, building on the heritage of systems currently in use on $8 \mathrm{~m}$ class telescopes. Recent advances in IR wavefront sensing have led to improved performance expectations for the GMT phasing system. Updated designs for the GMT acquisition, guiding, and wavefront sensing system (AGWS) and on-sky demonstrations of prototype phasing cameras are described in Section 5 of this paper and, in greater detail, elsewhere in these proceedings. ${ }^{12,15}$

Development of the site infrastructure for the GMT in Chile is nearly complete and excavations for the foundations of the enclosure and telescope pier will be underway in mid 2018.

As discussed in Section 7 below, and in other papers in these proceedings, ${ }^{16,17}$ we have conducted a thorough program of computational fluid dynamics (CFD) modeling of the airflow over the summit, and in and around the enclosure, to optimize its structure and location with respect to image quality. This has led to insights regarding the inflow of ground-layer air into the enclosure under a variety of lower enclosure geometries and locations on the summit platform. The increased height of the enclosure aperture and the primary mirror, compared to the $8 \mathrm{~m}$ telescopes, suggests that the GMT will be less susceptible to image degradation from uplift of warm air into the enclosure and the optical path of the telescope. This comprehensive modeling program has informed our understanding of the enclosure geometry and allowed us to make critical design decisions with confidence. The GMT site is among the most well-characterized astronomical locations on the planet, and its outstanding qualities are well documented.

In the sections that follow we describe the maturity of our project management and systems engineering approach, along with technical progress with the telescope structure and other key elements of the observatory.

\section{ORGANIZATION, REQUIREMENTS, AND PROCESS}

Figure 1 depicts the GMTO organization, with the project team indicated in blue. Cross-cutting functions, comprising systems engineering, project engineering, safety, assurance, and the project business office, are shown along the central vertical line. Eight deliverable subsystems are shown along the lower horizontal line. Each has a manager reporting to the project office, which includes the project manager, deputy project managers, and project scientist. Systems engineering is responsible for system-level architecture, requirements development and flow-down, configuration management and change control, verification \& validation, integrated modeling of system-level performance, technical budgets, product breakdown structure, interface control, and operations planning. Project engineering is responsible for system-level coordination of design, and for leading cross-cutting design tasks such as seismic engineering. The project business office interfaces with the corporate financial departments to implement project controls, including an earned value management system (EVMS) planned to begin in July 2018. Safety is responsible for environmental health and safety, design safety, and hazard analysis, while assurance is responsible for reliability, availability \& maintainability, technical standards, and quality. Three advisory bodies provide advice to the project office. They are the legacy advisory group of key designers who developed the reference design for the GMT, a management advisory council of experienced program managers, and a standing review board of subject matter experts. 


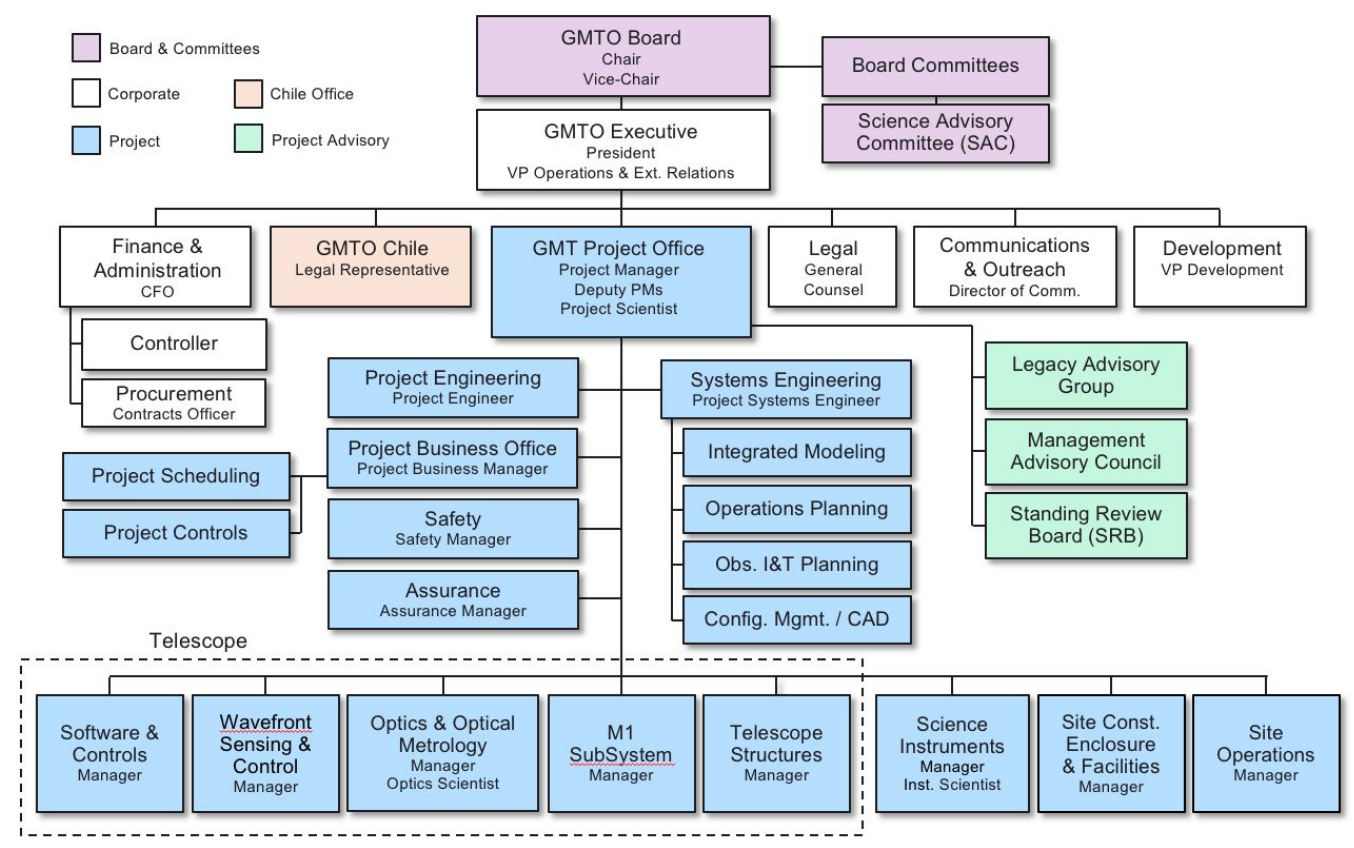

Figure 1. GMTO organization.

Science requirements have been revised and reorganized since the time of the 2014 design reviews in order to provide more complete performance requirements for all of the various observing modes of the telescope and clarify flowdown. Overall requirements organization is shown in Figure 2. The Observatory Requirements Document, Observatory Architecture Document, Concept of Operations document, and Operations Concepts document were reviewed in a System Requirements Review in April 2018.

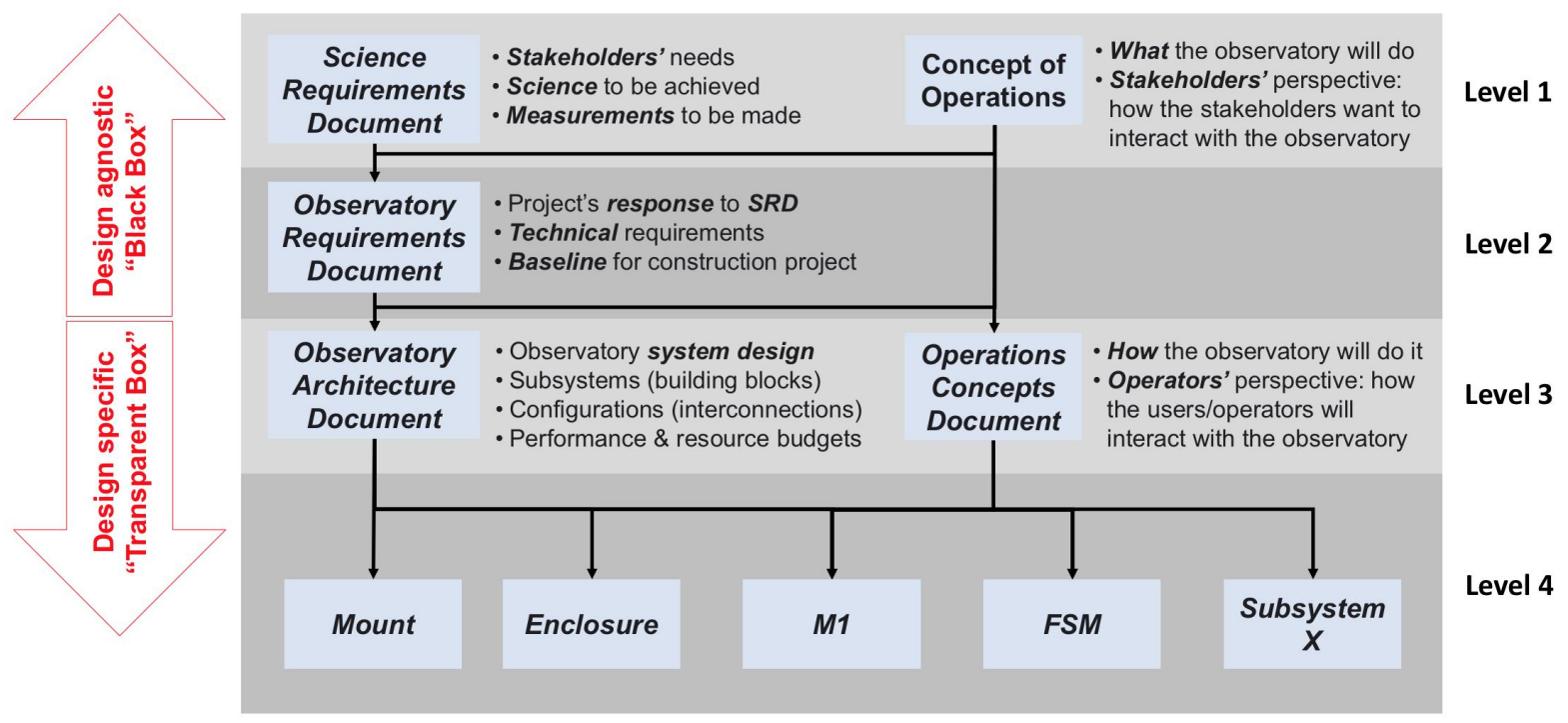

Figure 2. GMT requirements organization and flow down to Level-4 (subsystems).

All top-down technical budgets (both performance and resource) are well established, and most allocations are validated through integrated analysis and simulation. Major budgets include image quality for all wavefront control modes, 
pointing, throughput, emissivity, pupil and point spread function stability, distortion, target acquisition time, range of motion of optical elements, mass, utilities (power and cooling), maintenance and downtime, and seismic accelerations.

System level performance is estimated through simulations that link optical performance to technical specifications, perturbations, and environmental and operational conditions. To accomplish this, GMTO produced a powerful simulation framework that links high resolution optical models with structural, thermal, and controls models via an internally developed Dynamic Optical Simulation tool. This is augmented by in-house CFD analysis. ${ }^{16}$

\section{TELESCOPE DESIGN STATUS}

Papers in prior proceedings have described the design of the telescope mount, bearings, drives and other subsystems. ${ }^{18,19}$ The structure is a compact altitude-over-azimuth configuration using large $\mathrm{C}$-ring elevation bearings and a dual azimuth track. A third degree of motion is provided by the Gregorian instrument rotator, which performs image de-rotation for the various science instruments and houses the AGWS equipment. The design of the mount has not changed qualitatively since the 2014 design review. Modeling of the response of the telescope, primary mirror (M1), and secondary mirror (M2) subsystems to large seismic acceleration has, however, led us to implement seismic isolation in the telescope pier and to revisit the design of the M1 support system, particularly the force actuators and static supports.

Recent large earthquakes in Chile have led to more stringent seismic standards and a better characterization of the spectrum of ground accelerations experienced during a large seismic event. The site specific seismic hazard analysis was updated, adopting a probabilistic approach so that a fragility analysis could be performed for the M1 and M2 optics. Simulations revealed unacceptably large accelerations at the location of the azimuth track anchor bolts and at both M1 and M2. Modifications of the azimuth track structure and interface to the pier reduced the stresses in these locations to acceptable levels. The fragility analysis indicated that seismic base isolation is required to reduce accelerations at M1 and $\mathrm{M} 2$ to acceptable levels. ${ }^{20}$

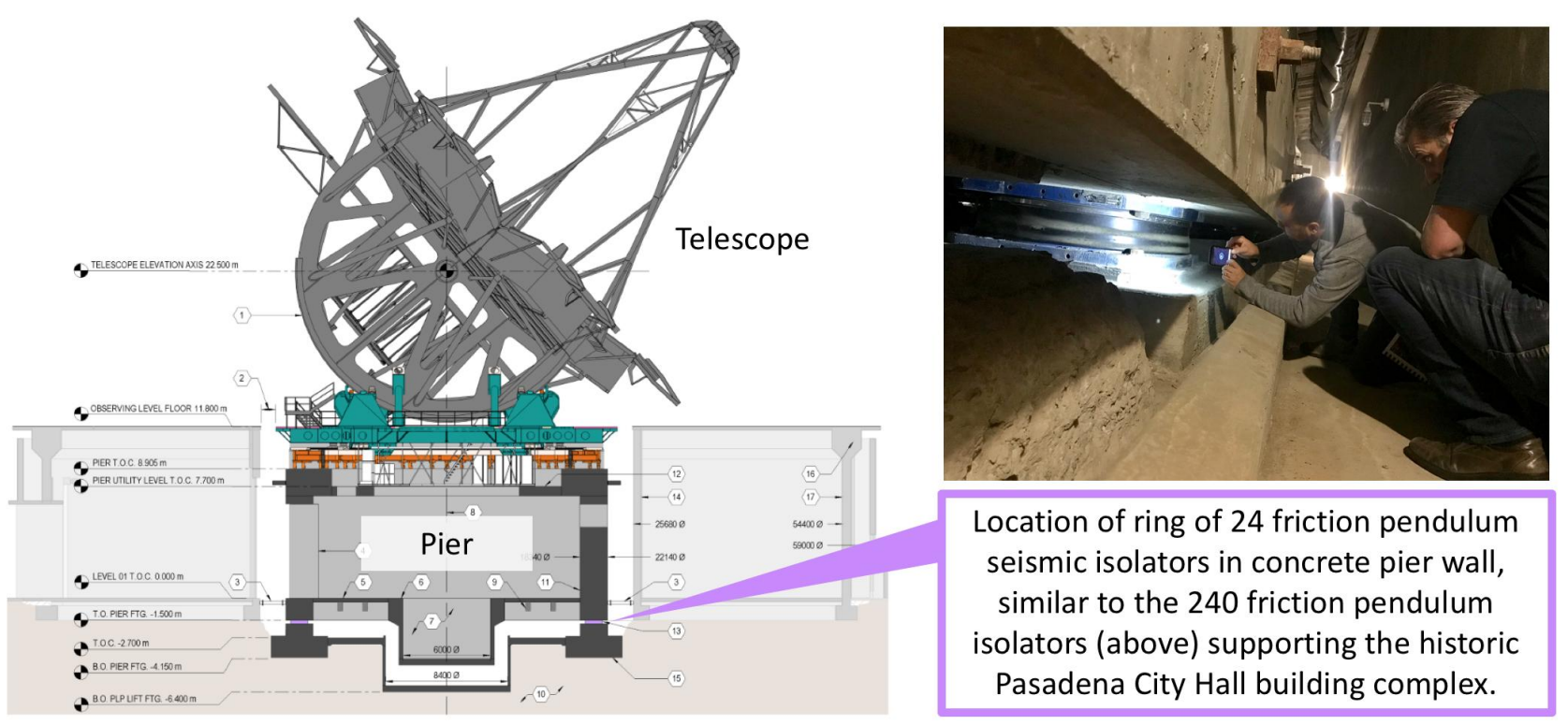

Figure 3. Cross-section of the telescope mount, pier and enclosure foundations. The seismic isolators are marked. They are similar to isolators supporting many civil engineering structures.

A variety of seismic isolation techniques were considered (e.g. lead-rubber dampers, double and triple friction pendulum isolators). The team settled on a set of single friction pendulum isolators as the best approach to reducing the accelerations in a survival level event while maintaining the stiffness of the mount during normal operations. ${ }^{21}$ Twentyfour single-friction pendulum devices are incorporated into the telescope pier ring wall and sized to permit the pier/telescope/mount combination to move laterally with respect to the ground by as much as $\pm 50 \mathrm{~cm}$. During a large 
seismic event the isolators will reduce accelerations at M1 and M2 by roughly $40 \%$. Mechanical actuators will be used to re-center the pier and isolators following an event large enough to displace the mount. Figure 3 illustrates the location of the friction pendulum isolators in the telescope pier ring wall.

GMTO presently has two teams under contract to advance the design of the mount prior to a down-select to a single design-build contractor. In the first stage of development the two contractor teams are reviewing the mount requirements, proposing changes to the reference design, analyzing the performance of the mount, and refining their cost estimates for the second stage of the procurement. We expect to have the stage 2 design-build team under contract in early 2019.

The GMT is a doubly-segmented optical system (Figure 4). Two secondary mirror systems are being developed, a conventional fast-steering mirror system and an adaptive secondary mirror system. The secondary mirror assemblies consist of seven $1.05 \mathrm{~m}$ segments, each aligned with its corresponding primary mirror segment. The functional requirements of the two secondary mirror systems and baseline designs have been described in prior reviews. ${ }^{22,23} \mathrm{We}$ have considered two approaches to the interface between the secondary mirrors and the telescope. The first is a singlestage interface consisting of one large stroke hexapod per segment that provides both positioning and tip-tilt control. In the alternate design, a stiff macro-cell interfaces to the truss through a large-stroke hexapod, which provides overall position control of the secondary mirror array relative to a rigid reference, while a set of short-stroke hexapods provides individual position control and tip-tilt correction for each segment (Figure 4). Separating the position control between the two stages reduces the range of motion for the small hexapods and thereby eliminates the risk of M2 segment collision. A trade study is presently underway that will lead to a selection of the support interface in the third quarter of 2018.
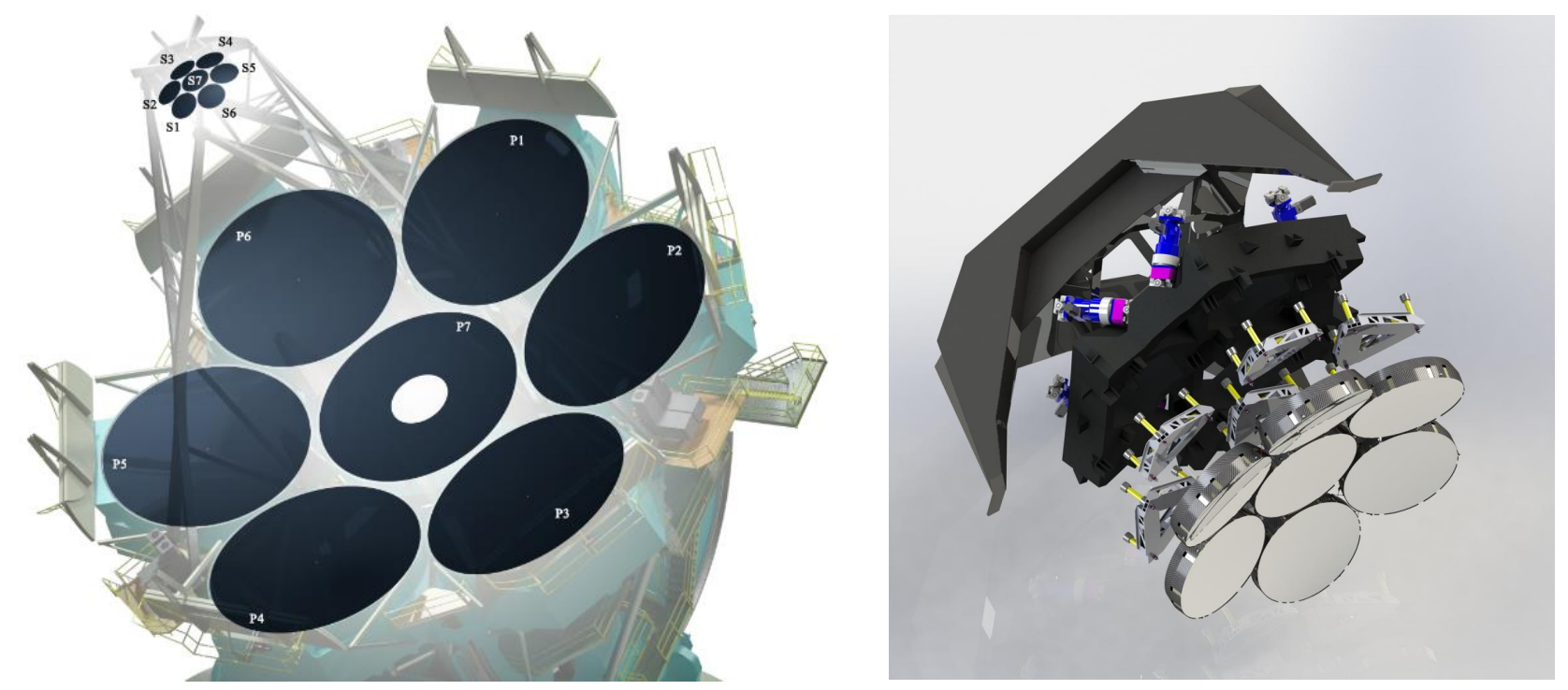

Figure 4. The doubly-segmented structure and conjugation of the GMT primary (M1) and secondary (M2) optics (left). The twostage architecture of the fast-steering secondary mirrors (right) separates rigid body motion from the fine positioning and tip-tilt of the individual segments.

\section{PRIMARY MIRROR PRODUCTION STATUS}

The primary mirror segments continue in production at the University of Arizona's Richard F. Caris Mirror Lab. They have the longest development timeline of all of the project elements. Martin and colleagues have documented the process used to fabricate the mirror blanks and to figure the off-axis mirrors to the required surface specifications. At this time Segment 1 (an off-axis mirror) is complete and has been placed in storage. Mirror Segments 2, 3, 4, and 5 are currently in production (Segment 4 being the center, on-axis mirror). Long-lead material procurements have been made for mirror Segments 6 and 7. 
Martin et al. describes a number of process improvements that the mirror lab team is using to figure Segment $2 .^{7}$ These are expected to reduce risk and processing time compared to Segment 1 by using more up-to-date tools and technology in the metrology and polishing steps.

Primary mirror Segment 2 is in the final figuring stage. Mirror Segment 3 is undergoing front surface generating with diamond tooling. Rear surface polishing of Segment 4 is now complete and load spreaders are being attached for use in the polishing stage and for mounting in the mirror cells. Segment 5 was cast in November of 2017; the refractory material has been cleaned out and inspection is in progress. The status of each of the segments is summarized in Table 1.

Table 1. GMT primary mirror development status.

\begin{tabular}{|c|l|l|}
\hline Segment & \multicolumn{1}{|c|}{ Status } & \multicolumn{1}{c|}{ Notes } \\
\hline 1 & Complete & In storage off site from mirror lab \\
\hline 2 & Front surface figuring & On Large Polishing Machine \\
\hline 3 & Front surface generating & On Large Optics Grinder \\
\hline 4 & Load spreader attachment & Center mirror segment \\
\hline 5 & Cast blank inspection & Cast in Nov 2017 \\
\hline 6 & Awaiting casting & Ohara E-6 glass in hand \\
\hline 7 & Awaiting casting & Ohara E-6 glass in hand \\
\hline
\end{tabular}

\section{ADAPTIVE OPTICS AND WAVEFRONT CONTROL}

The GMT will operate in one of four observing modes depending on the field of view, image quality, and sky coverage needs of the scientific program. ${ }^{12}$ These are: 1) natural seeing, 2) ground-layer adaptive optics (GLAO), 3) natural guidestar adaptive optics (NGAO), and 4) laser tomography adaptive optics (LTAO). Significant effort has been focused recently on increasing the fidelity of simulations of these observing modes and developing flow-down requirements on telescope subsystems and control algorithms. The doubly-segmented optical design and actively-controlled M1 and M2 (adaptive secondary mirror) segment figure result in a significant number of blind and degenerate optical modes of the system. These can nevertheless be controlled by combining the measurements of off-axis wavefront sensors of the AGWS with on-axis adaptive optics wavefront sensors in the diffraction-limited NGAO and LTAO modes. ${ }^{24}$ The control of segment phasing similarly requires simultaneous wavefront measurements at three field positions, and high-bandwidth feedback from M1 and M2 edge sensors. ${ }^{25}$ Our active and adaptive optics simulations incorporate dome seeing and wind pressures derived from CFD analysis, ${ }^{16}$ and the structural dynamics of the mount and optics. ${ }^{9}$

The AGWS provides all necessary wavefront sensing to implement the natural seeing and GLAO observing modes, and feedback to maintain the phase piston alignment of the GMT segments. ${ }^{15}$ The AGWS passed its Preliminary Design Review (PDR) in June 2017 and is now in the detailed design phase. It consists of four independent probes which patrol the periphery of the Direct Gregorian field of view (Figure 5). Each probe includes a visible Shack-Hartmann wavefront sensor for guiding, active optics, and GLAO wavefront sensing, along with an infrared dispersed fringe sensor that allows determinations of piston errors between the GMT segments. A prototype dispersed fringe sensor was tested on the Magellan Clay telescope in May 2018 (Figure 6). ${ }^{26}$ The adaptive optics wavefront sensors passed PDR in 2013. 

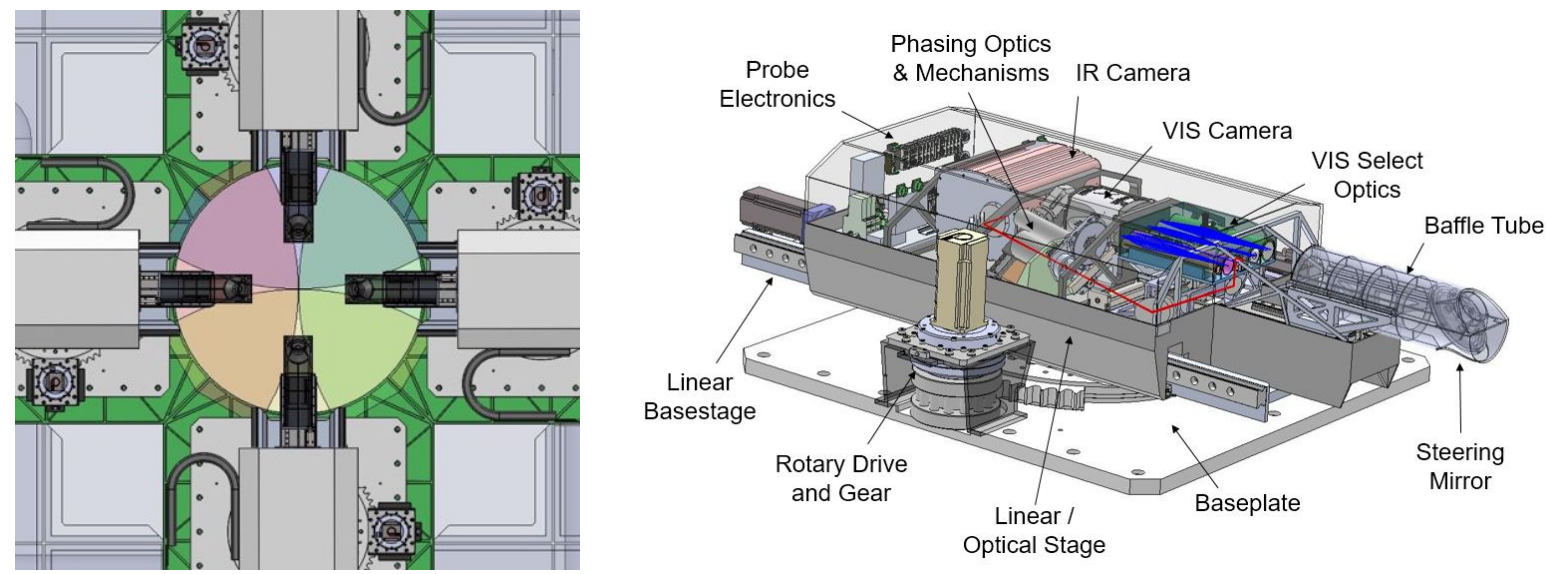

Figure 5. Preliminary design of the AGWS. (Left) Patrol ranges of the four AGWS probes as seen from the direct Gregorian instrument. (Right) AGWS probe detail.

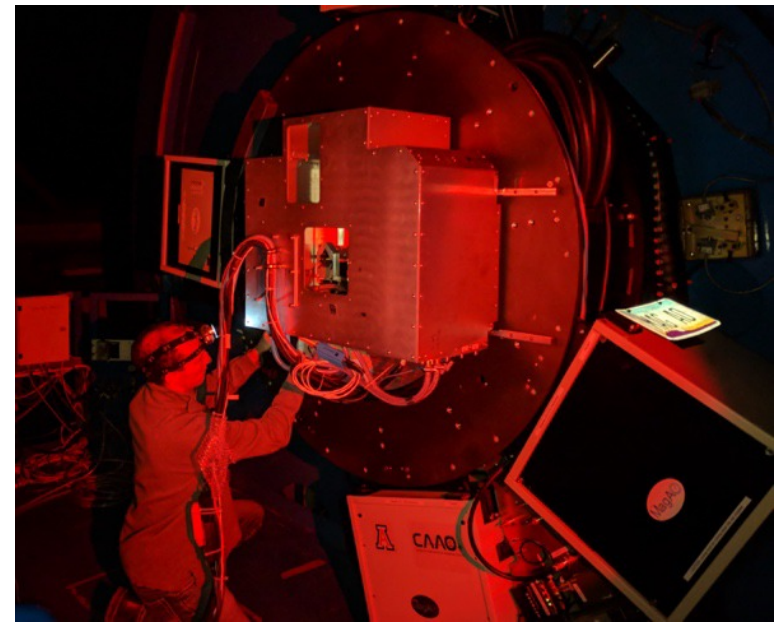

Figure 6. The AGWS infrared dispersed fringe sensor prototype on the 6.5m Magellan Clay telescope, May 2018.

\section{SCIENTIFIC INSTRUMENTS}

Up to eleven scientific instruments can be accommodated on the telescope at various stations, as illustrated in Figure 7. A set of first generation instruments was identified through an open solicitation for concepts early in the preliminary design phase. Concepts considered and instruments under development have been reported in prior SPIE conferences. ${ }^{24}$ The instruments under development at this time include high resolution spectrographs (visible and IR), a visible-light multi-object spectrograph and an AO-fed combination imager and integral field spectrograph. These instruments are at different levels of maturity as they are planned to be commissioned on the telescope at different times. We will not report on all of these instruments in this overview as they are covered in other reports in these proceedings. ${ }^{27,28,29,30,31}$

The G-CLEF (GMT-Consortium Large Earth Finder) high-resolution optical spectrograph is the most mature GMT instrument at this time. ${ }^{27}$ This instrument is being developed by a team at the Harvard-Smithsonian Center for Astrophysics with collaborators at several other institutions. It is targeted towards exoplanet research but will also enable fundamental work in stellar astrophysics, stellar and interstellar chemistry, galaxy evolution, and cosmology. It builds on the HARPS North precision radial velocity spectrograph deployed on the Galileo National Telescope. ${ }^{32}$ A rendering of the G-CLEF spectrograph inside its vacuum enclosure is shown in Figure 8. 

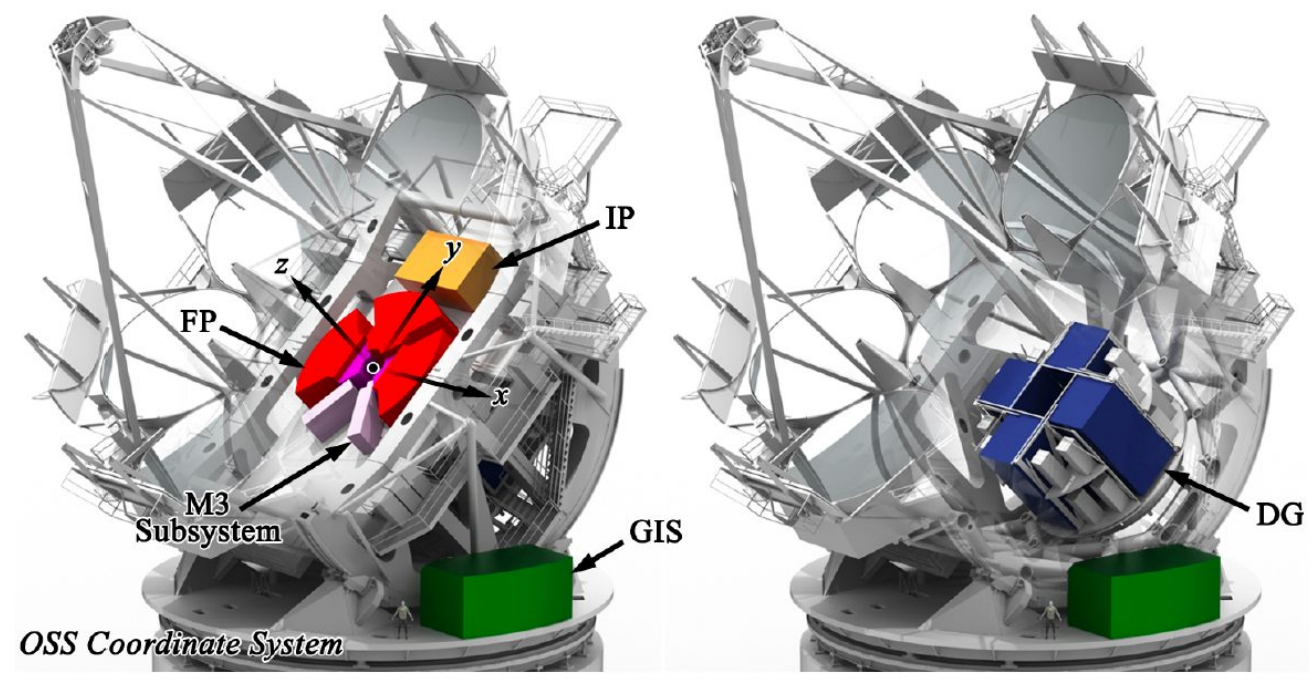

Figure 7. GMT instrument stations include four direct Gregorian (DG); one gravity invariant station (GIS); three folded port (FP), one instrument platform (IP); and two auxiliary port (AP; not shown), which are also gravity invariant and located on either end of the elevation axis.
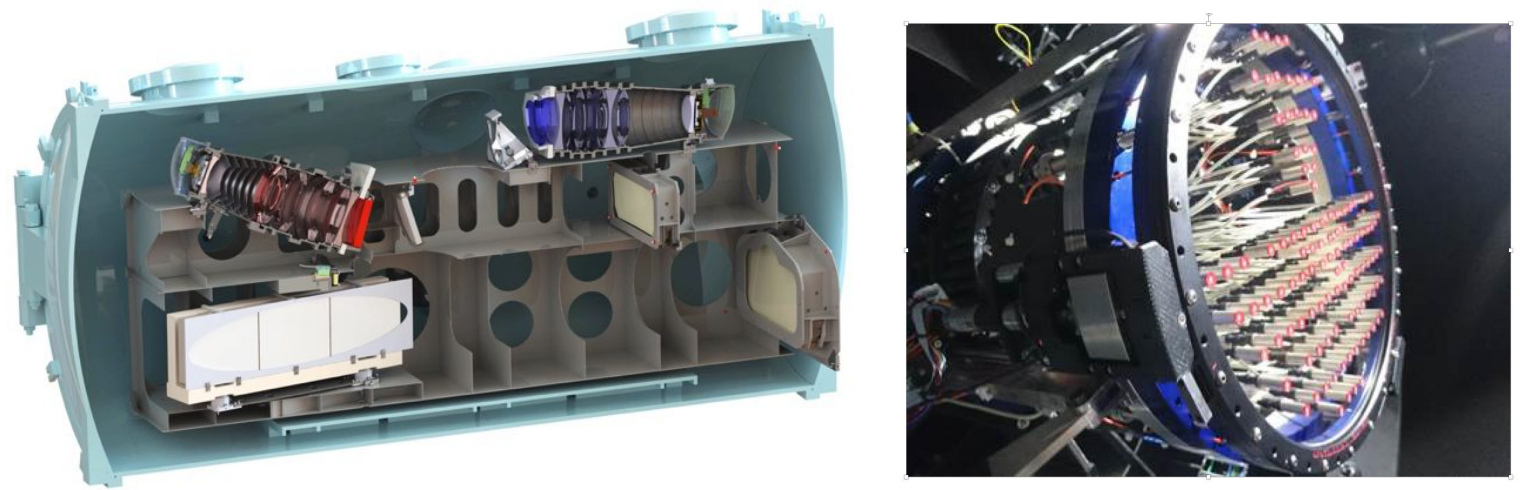

Figure 8. Left: G-CLEF spectrograph inside its vacuum enclosure ( 4.3m x $2.1 \mathrm{~m}$ x $2.3 \mathrm{~m})$. Right: The TAIPAN fiber positioner on the UK Schmidt Telescope at Siding Spring Observatory, shown with $\sim 100$ of its 150 Starbugs, capable of delivering optical fibers to any position within the 6-degree diameter field of view to an accuracy of 5 microns.

The principal faint object/survey spectrograph for the GMT is GMACS (GMT Multi-object Astronomical and Cosmological Spectrograph), which is being developed by a team at Texas A\&M University. ${ }^{28}$ Its architecture is based on a single spatial channel with dual red and blue cameras. The power of the G-CLEF and GMACS spectrographs can be enhanced by means of the GMT facility fiber system MANIFEST (Many Instrument Fiber System). ${ }^{31}$ Self-motile fiber heads, called "Starbugs," patrol the focal plane on a curved glass plate, enabling a massively-multiplexed rapidlyreconfigurable fiber positioner capability. An on-sky demonstrator system called TAIPAN is currently being commissioned on the UK Schmidt telescope at Siding Spring Observatory, as shown in Figure 8.

\section{ENCLOSURE DESIGN}

The GMT enclosure design has continued to evolve since 2016, based on a series of engineering trade studies, CFD analyses, site wind flow measurements, and cost assessments. ${ }^{16,33,34,35}$ In the interest of simplicity and operational reliability, a conventional bi-parting shutter door has been adopted. This design necessitates separate wind and moon screens, which can be optimized individually for wind control and stray light protection in the observing chamber. 
Considerable attention has been paid to the lower (non-rotating) portion of the enclosure. The 6.5-m Magellan telescopes at the Las Campanas Observatory feature "open-soffit" lower enclosures, a design feature that allows wind-driven air to flow freely under the observing floor, which may reduce uplift of ground-heated air into the upper enclosure where it can negatively impact image quality. This feature is believed to contribute to the excellent image quality demonstrated by the Magellan telescopes, and was carried over into the preliminary design of the lower enclosure for the GMT. However, there are many advantages to a more conventional "closed" lower enclosure design, including improved structural rigidity and seismic strength, creation of usable (and valuable) interior space, and lower cost. We therefore undertook comparative CFD studies of the open and closed soffit lower enclosure designs (Figure 9), as well as a hybrid open/closed design having a perforated ring wall. Analysis thus far indicates virtually no meaningful difference in predicted image quality contributions between these three configurations. This appears to be due to the height of the GMT telescope and the enclosure viewing aperture above the ground - much higher than Magellan - and much higher than the size of the stagnation vortex that is generated at the base of the closed soffit. ${ }^{16}$ Ventilation and control of airflow through the upper enclosure is critical to mitigating "dome seeing." ${ }^{36}$ Thermal CFD modeling of the observatory, including telescope, enclosure, and enclosure features such as wind vents, wind screen, shutter doors, and moon screen will continue during the final design phase in 2019.

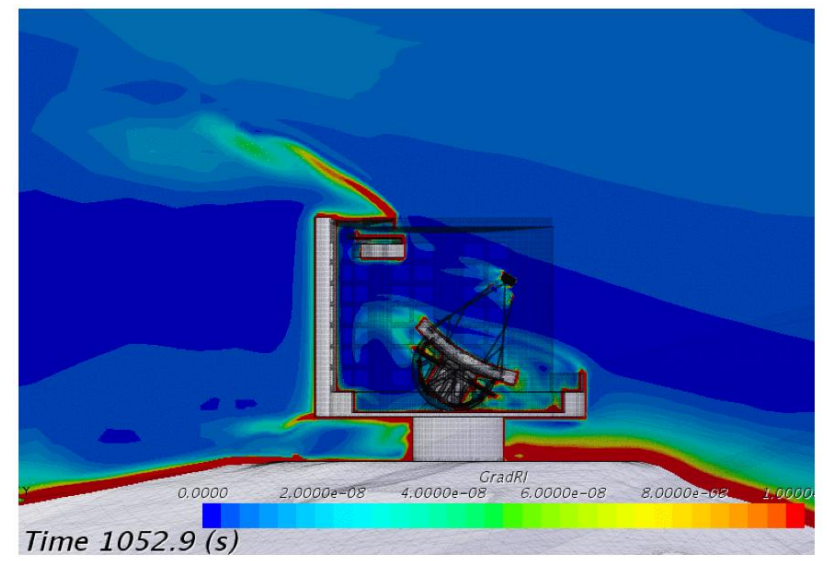

a) Open soffit lower enclosure.

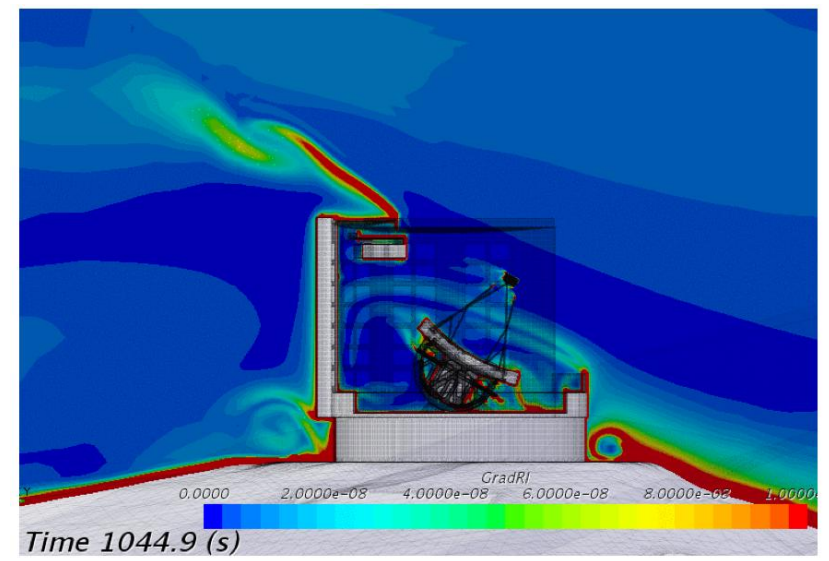

b) Closed soffit lower enclosure.

Figure 9: Comparison of refractive index gradients in unsteady air flow for open and closed soffit lower enclosure architectures. Note the stagnation vortex in the closed soffit case.

\section{OBSERVATORY CONTROL SYSTEM}

The Observatory Control System (OCS) includes all software and hardware components necessary to control and monitor the various optical and electromechanical subsystems, and to safely and efficiently operate the observatory. The OCS architecture (Figure 10) follows a component-based model approach, where components are specified using Domain Specific Language created for this purpose. This enables code generation in several languages and automatic validation of architectural conformance and interfaces. Core components have been defined and initial implementations now exist. A software development kit, needed to build applications, is delivered to external teams every six months. The Q2 2018 kit contains the first frameworks and services to support the build of telescope Device Control Subsystems (DCS).

OCS development follows Agile development methods ${ }^{37}$ To assure adequate development formalism and traceability, requirements and user-stories are being developed and linked to acceptance criteria that can be mapped to formal verification and validation methods. We are following NDIA guidelines to map methods and artifacts to our EVMS, ${ }^{38}$ and are piloting this on a portion of the Work Breakdown Structure (WBS). 


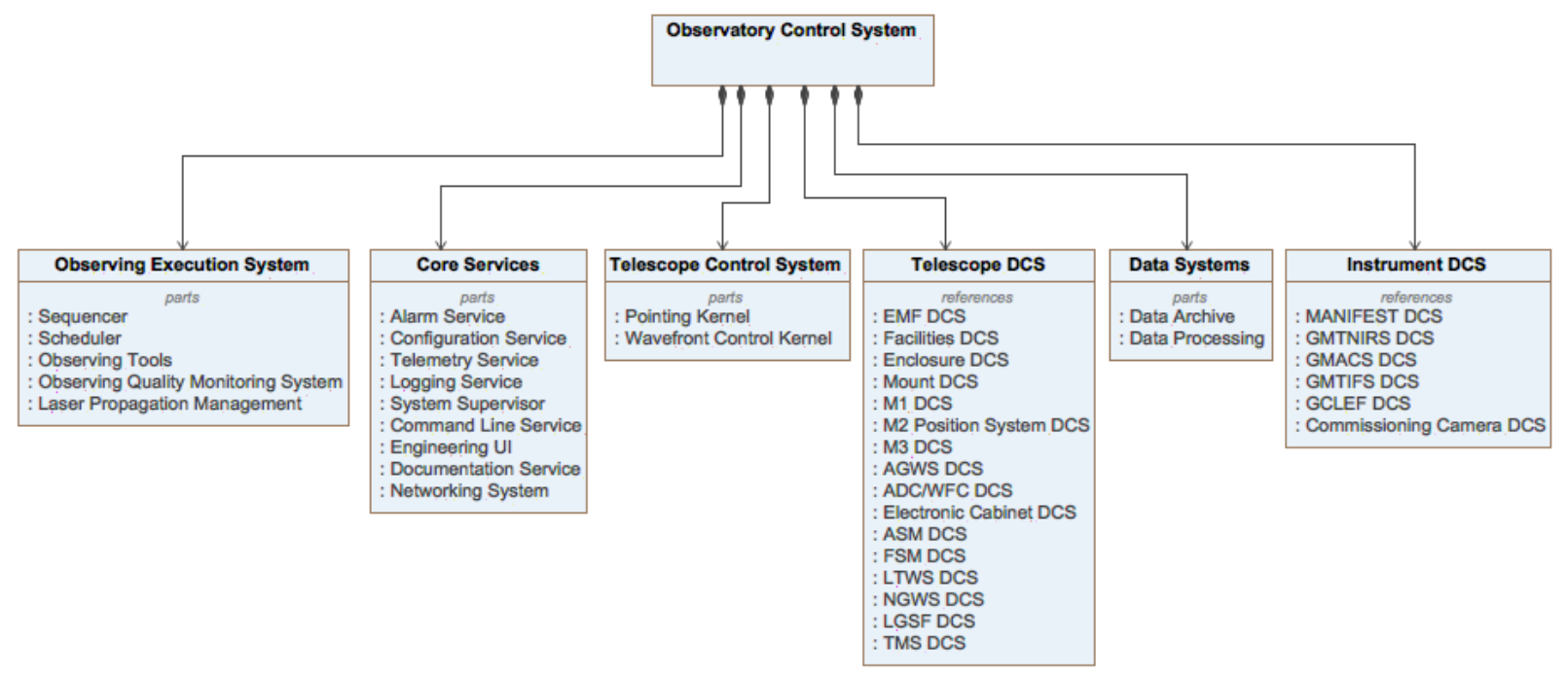

Figure 10. Observatory control system architecture.

\section{SITE CONSTRUCTION AND PLANS}

The GMT is being built at the Las Campanas Observatory in northern Chile. The site is owned by the Carnegie Institution for Science and is home to the twin Magellan $6.5 \mathrm{~m}$ telescopes and a number of smaller instruments. The site was leveled in 2012 and construction on the summit has been underway since that time. The site master plan is shown in Figure 11. The location of the enclosure has been moved to the South-East end of the summit mesa based on CFD analysis indicating a small air flow advantage. A summit support building dedicated to primary mirror coating has been placed adjacent to the enclosure. The master plan is otherwise unchanged from the last overview paper in $2016,{ }^{6}$ although considerable new infrastructure has been completed at the site since that time. Figure 12 shows a rendering of the summit site and Figure 13 shows a recent photograph.

New infrastructure on the summit mesa includes construction offices with a conference room and a first aid station, a MASS/DIMM site-seeing monitor, and new electrical construction power distribution. At the time of writing (April 2018), drilling of micro-piles for the enclosure foundations is underway and the start of hard rock excavation for the telescope and enclosure foundations is imminent. Start of construction of the concrete foundations for the telescope and enclosure is planned for mid-2019.

Support site no. 2 is shown in Figure 14. Completed infrastructure at support site no. 2 includes a 68-room residence building capable of housing a construction crew of 200 persons, a 24-room residence for GMTO employees and visitors, and dining and recreation facilities. In addition to electrical power, site infrastructure includes backup power generators, wired and wireless internet, potable and firefighting water, and waste water treatment. Ultimately, electrical capacity will need to increase from construction power to an estimated 5MW for operations. GMTO is exploring options for routing a future new power line to the site for this purpose. 


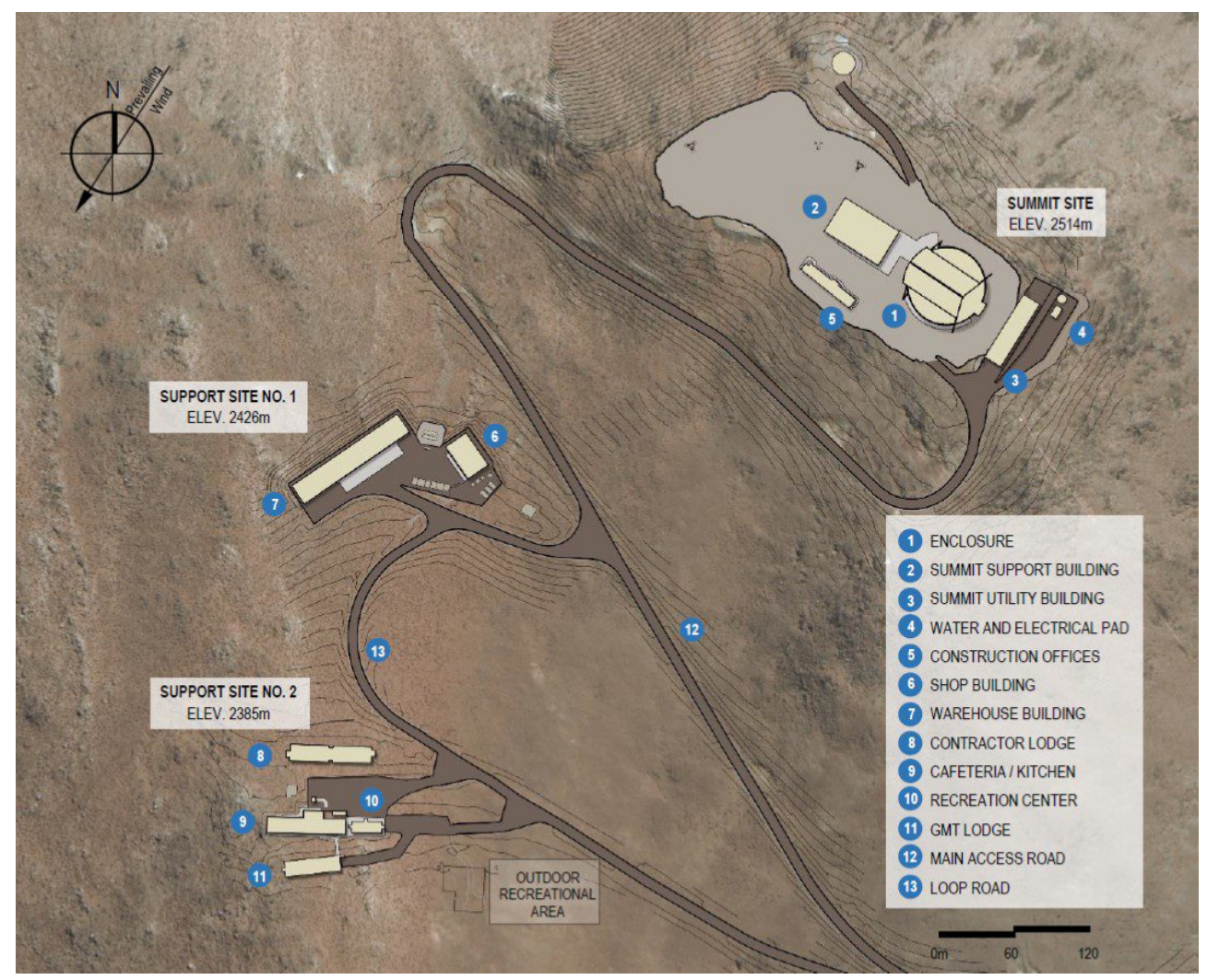

Figure 11. The GMT site master plan showing the summit site mesa, support site no. 1 dedicated to workshops and laboratories, and support site no. 2 dedicated to residences, dining, and recreation facilities.

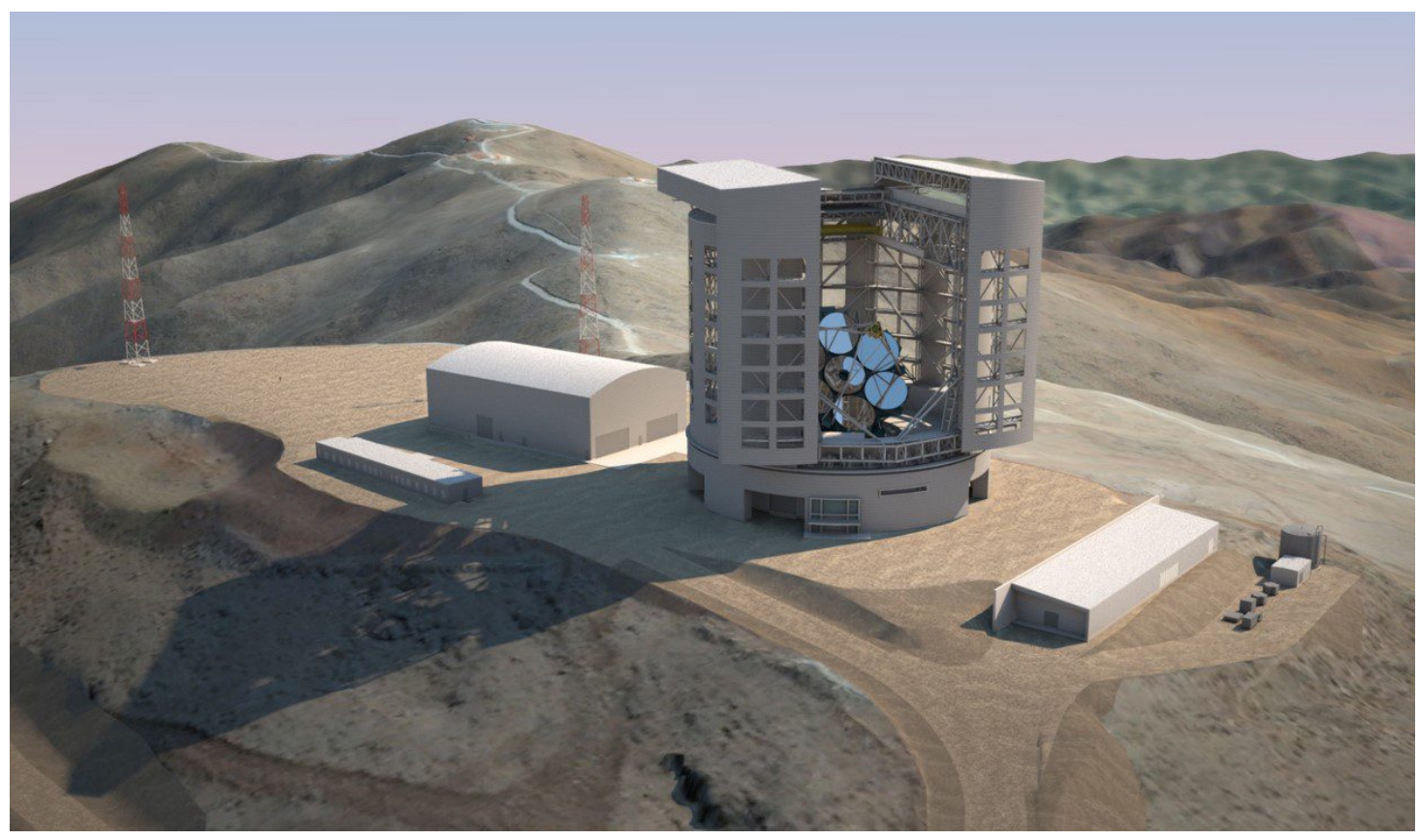

Figure 12. Rendering of the GMT summit site with (left to right) the summit support building, enclosure (hybrid perforated ring wall variant), summit utility building, and water storage and distribution equipment. 


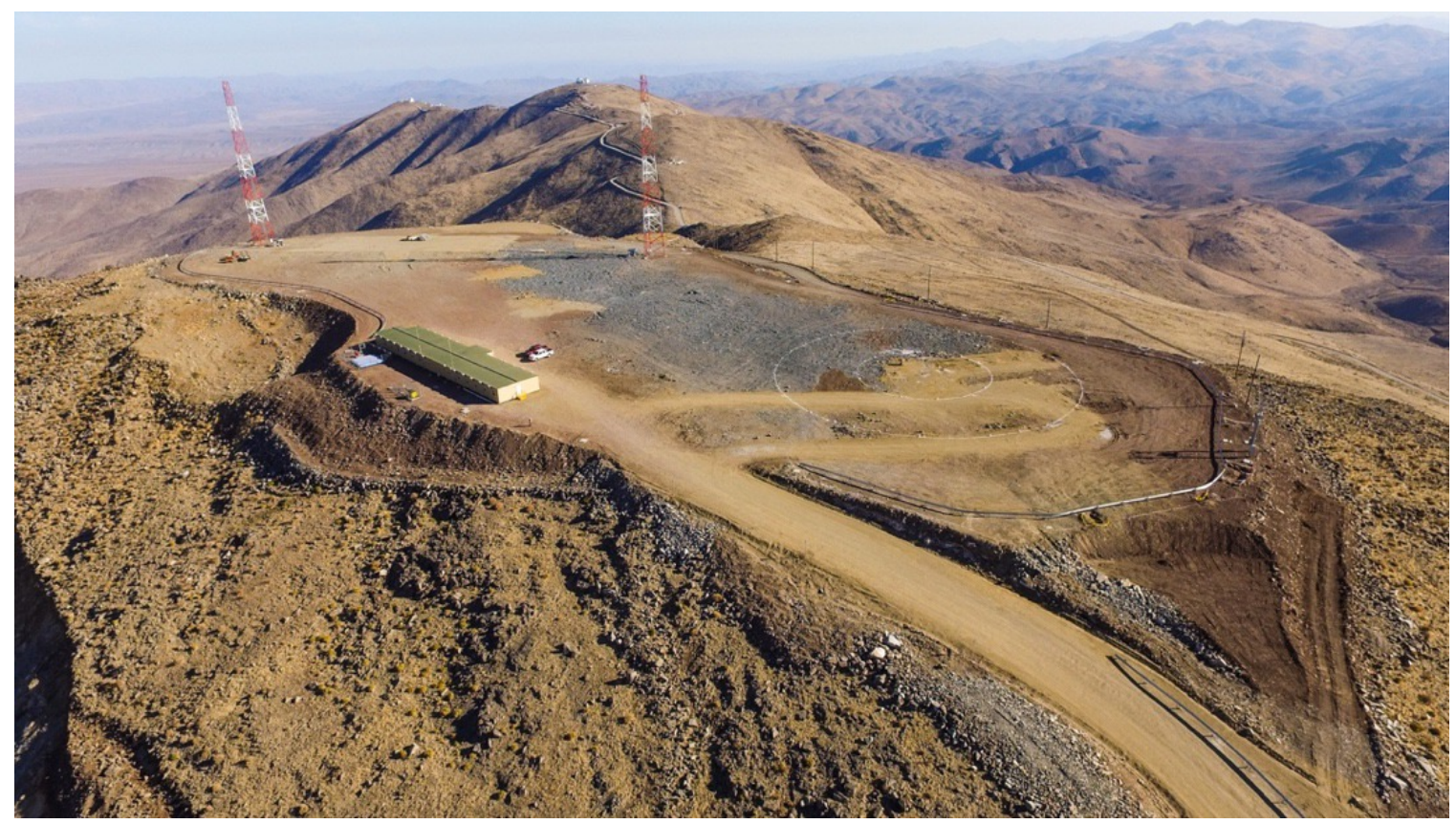

Figure 13. View of the summit site mesa looking North toward the Las Campanas Observatory. Construction offices are seen on the left. Concentric white circles (enclosure and pier concrete ring wall diameters) indicate the future location of the GMT.
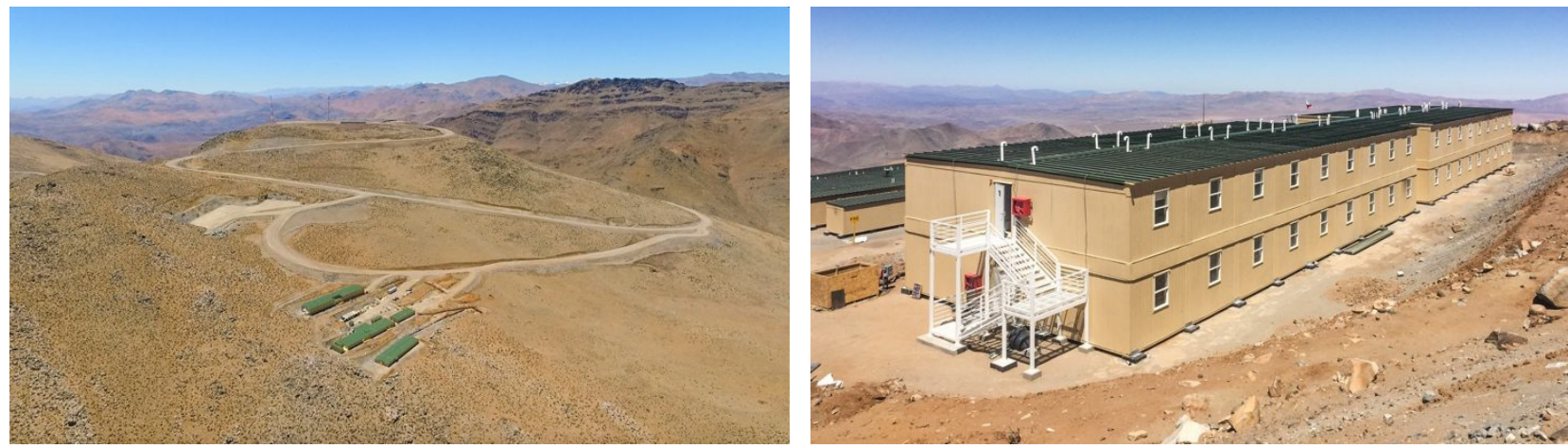

Figure 14. Left: Support site no. 2 showing residence, dining and recreation facilities ready to support construction. The leveled summit site can be seen above support site no. 2. Right: Construction workers' residence accommodates a crew of 200.

\section{PROJECT STAGING AND SCHEDULE}

As discussed in previous overviews, the GMT project is structured in multiple stages. The staging allows us to match the project schedule and scope with funding commitments and to simplify management of the schedule critical path. The development time-line for the primary mirror segments is longer than that of the conventional construction and the major mechanical systems. Our schedule shows that the mount will be ready to receive the primary optics at a time when we expect to have fewer than seven mirrors in Chile. Integrating these first mirror segments into their cells and then into the telescope prior to the arrival of the remaining segments will speed the overall project completion and will allow us to commission key aspects of the observatory sooner than would otherwise be possible. 
Similarly, our schedule calls for the conventional secondary mirrors to be ready more than two years before the full set of adaptive secondary mirrors are on site. In the first stage of deployment, we will use the conventional secondary mirrors to commission the active optics and telescope metrology systems before moving to the full adaptive optics operations. This allows us to get on the sky sooner and to reduce the complexity of the early commissioning phases.

The overall project schedule has stretched somewhat compared to our 2016 update. We have focused on keeping the most critical items as close to the original schedule as possible. Site acceptance of the mount and delivery of primary mirror Segment 2 have moved by three calendar quarters. Completion of the enclosure is off the critical path and we expect that our completion of the site infrastructure and deployment of the construction management team to Chile will keep this work on schedule. Schedule elements that are less critical to completion of the facility are being managed to ensure that they arrive on the site when needed and do not create resource conflicts with critical path elements.

\section{SUMMARY}

The Giant Magellan Telescope project is progressing with design, fabrication, and site construction. The Caris Mirror Lab is now processing four primary mirror segments and materials are on hand for the remaining two segments. We are engaged with industry to complete the design of the telescope structure and will soon down-select to a single design-build team. Construction activities on the summit site are well underway and the rate of activity will continue to accelerate over the coming year. The systems engineering foundations of the project have been strengthened over the past two years and these will support the final design of key subsystems, major procurements, verification and validation of subsystems, and integration of the observatory.

This work has been supported by the GMTO Corporation, a non-profit organization operated on behalf of an international consortium of universities and institutions: Arizona State University, Astronomy Australia Ltd, the Australian National University, the Carnegie Institution for Science, Harvard University, the Korea Astronomy and Space Science Institute, the São Paulo Research Foundation, the Smithsonian Institution, the University of Texas at Austin, Texas A\&M University, the University of Arizona, and the University of Chicago.

\section{REFERENCES}

[1] Johns, M., "The Giant Magellan Telescope (GMT)," Proc. SPIE 6267, Ground-based and Airborne Telescopes, 626729 (2006).

[2] Johns, M., "Progress on the GMT," Proc. SPIE 7012, Ground-based and Airborne Telescopes II, 70121B (2008).

[3] Shectman, S.; Johns, M., "GMT overview," Proc. SPIE 7733, Ground-based and Airborne Telescopes III, 77331Y (2010).

[4] Johns, M. et al., "Giant Magellan Telescope: overview," Proc. SPIE 8444, Ground-based and Airborne Telescopes IV, 84441H (2012).

[5] Bernstein, R. A. et al., "Overview and status of the Giant Magellan Telescope project," Proc. SPIE 9145, Groundbased and Airborne Telescopes V, 91451C (2014).

[6] McCarthy, P. et al., "Overview and status of the Giant Magellan Telescope project," Proc. SPIE 9906, Ground-based and Airborne Telescopes VI, 990612 (2016).

[7] Martin, H. et al., "Manufacture of the primary mirror segments for the Giant Magellan Telescope," Proc. SPIE 10706, Advances in Optical and Mechanical Technologies for Telescopes and Instrumentation III, 10706-30 (2018).

[8] Angeli, G. et al., "Systems engineering for the Giant Magellan Telescope," Proc. SPIE 10705, Modeling, Systems Engineering, and Project Management for Astronomy VIII, 10705-17 (2018).

[9] Irarrazaval, B. et al., "The Giant Magellan Telescope integrated modeling and performance," Proc. SPIE 10705, Modeling, Systems Engineering, and Project Management for Astronomy VIII, 10705-34 (2018).

[10] Ranka, T. et al., "Performance analysis tools and results for Giant Magellan Telescope primary mirror segments active support system," Proc. SPIE 10705, Modeling, Systems Engineering, and Project Management for Astronomy VIII, 10705-35 (2018).

[11]Bigelow, B. et al., "GMT site, enclosure, and facilities: design and construction update," Proc. SPIE 10700, Groundbased and Airborne Telescopes VII, 10700-8 (2018). 
[12] Bouchez, A. et al., "An overview and status of GMT active and adaptive optics," Proc. SPIE 10703, Adaptive Optics Systems VI, 10703-33 (2018).

[13]Filgueira, J., et al., "Status of the observatory control system for the GMT," Proc. SPIE 10707, Software and Cyberinfrastructure for Astronomy V, 10707-4 (2018).

[14]Dressler, A. et al., "IMACS: the wide-field imaging spectrograph on Magellan-Baade," Proc. SPIE 6269, Groundbased and Airborne Instrumentation for Astronomy, 62690F (2006).

[15] McLeod, B., "The acquisition, guiding, and wavefront sensing system for the Giant Magellan telescope," Proc. SPIE 10700, Ground-based and Airborne Telescopes VII, 10700-60 (2018).

[16] Vogiatzis, K., et al., "Computational fluid dynamics modeling of GMT," Proc. SPIE 10705, Modeling, Systems Engineering, and Project Management for Astronomy VIII, 10705-28 (2018).

[17]Das, K. et al., "GMT aerothermal modeling validation through site measurements," Proc. SPIE 10705, Modeling, Systems Engineering, and Project Management for Astronomy VIII, 10705-2 (2018).

[18] Johns, M. et al., "Design of the Giant Magellan Telescope" Proc. SPIE 9145, Ground-based and Airborne Telescopes $\mathrm{V}, 91451 \mathrm{~F}(2014)$.

[19] Sheehan, M. et al., "Progress on the structural and mechanical design of the Giant Magellan Telescope," Proc. SPIE 8444, Ground-based and Airborne Telescopes IV, 84440N (2012).

[20] Irarrazaval, B. et al., "Minimization of seismic risk at the Giant Magellan telescope," Proc. SPIE 10700, Groundbased and Airborne Telescopes VII, 10700-108 (2018).

[21] Manual, E. et al., "Telescope pier seismic isolation for the Giant Magellan telescope," Proc. SPIE 10700, Groundbased and Airborne Telescopes VII, 10700-222 (2018).

[22] Cho., M. et al., "Development of GMT fast steering secondary mirror assembly," Proc. SPIE 9145, Ground-based and Airborne Telescopes V, 91451M (2014).

[23] Biasi, R. et al., "GMT adaptive secondary design," Proc. SPIE 7736, Adaptive Optics Systems II, 77363 (July 2010).

[24] Conan, R. et al., "Control algorithms and performance of the active optics system of the Giant Magellan Telescope", Proc. SPIE 10700, Ground-based and Airborne Telescopes VII, 10700-110 (2018).

[25] Quirós-Pacheco, F. et al., "The Giant Magellan Telescope phasing strategy and performance", Proc. SPIE 10700, Ground-based and Airborne Telescopes VII, 10700-18 (2018).

[26] Kopon, D. A. et al., "On-sky results of the next generation GMT phasing sensor prototype", Proc. SPIE 10703, Adaptive Optics Systems VI, 10703-34 (2018).

[27] Szentgyorgyi, A. et al., "The GMT-consortium large earth finder (G-CLEF): an optical echelle spectrograph for the Giant Magellan telescope (GMT)," Proc. SPIE 10702, Ground-based and Airborne Instrumentation for Astronomy VII, 10702-63 (2018).

[28]DePoy, D. et al., "The Giant Magellan telescope multi-object astronomical and cosmological spectrograph (GMACS)," Proc. 10702, Ground-based and Airborne Instrumentation for Astronomy VII, 10702-69 (2018).

[29] Kidder, B. et al., "Manufacturing silicon immersion gratings for GMTNIRS on 150-mm material," Proc. 10703 Advances in Optical and Mechanical Technologies for Telescopes and Instrumentation III, 10706-73 (2018).

[30] Sharp, R. et al., "Design evolution of the Giant Magellan telescope integral field spectrograph, GMTIFS," Proc. SPIE, Ground-based and Airborne Instrumentation for Astronomy VII, 10702-67 (2018).

[31] Lawrence, J. et al., "Wide-field multi-object spectroscopy with MANIFEST," Proc. SPIE, Ground-based and Airborne Instrumentation for Astronomy VII, 10702-372 (2018).

[32] Cosentino, R. et al., "Harps-N: the new planet hunter at TNG," Proc SPIE 8446, Ground-based and Airborne Instrumentation for Astronomy IV, 84461V (2012).

[33] Teran, J. et al., "GMT site: facilities and enclosure design overview," Proc. SPIE 9145, Ground-based and Airborne Telescopes V, 91452K (2014).

[34] Bigelow, B. et al., "GMT site, enclosure, and facilities: design and construction update," Proc. SPIE, Ground-based and Airborne Telescopes VII, 10700-8 (2018).

[35] Khodadoust, A. et al., "Giant Magellan telescope site and enclosure CFD modeling and analysis," Proc. SPIE 10705, Modeling, Systems Engineering, and Project Management for Astronomy VIII, 10705-1 (2018).

[36] Bauman, S. et al., "Dome venting: the path to thermal balance and superior image quality," Proc. SPIE 9149, Observatory Operations: Strategies, Processes, and Systems V, 91491K (2014).

[37]Beck, K. et al., "Manifesto for Agile Software Development," http://agilemanifesto.org (15 May 2018).

[38][An Industry Practice Guide for Agile on Earned Value Management Programs], National Defense Industrial Association (NDIA) Integrated Program Management Division (IPMD), Ver. 1.1 (2017). 